\title{
Crown-root fracture management by therapeutic extraction and intentional replantation in children
}

\begin{abstract}
Intentional replantation is in practice from past many years as a treatment option for pulpless teeth and also an effective treatment option for teeth which are difficult or impossible to treat using the conventional root canal therapy. The preservation of periodontal ligament vitality, the removal of all tissue debris and irritating substances from the root surface, establishment of a good apical seal and reinforcement of the crown structure are crucial in establishment of the normal function of the reimplanted tooth. This procedure should be considered as an alternative to tooth extraction. A case of vertical crown root fracture of a permanent maxillary central incisor treated with intentional replantation is reported in this article. At the two-year recall visit, the patient was asymptomatic. The tooth was still in function and radiograph showed no evidence of pathological changes.
\end{abstract}

Keywords: ankylosis, intentional replantation, periodontal ligament, therapeutic extraction
Volume 6 Issue 5 - 2017

\author{
Anil T Patil, Ankita R Hingmire, Sachin A \\ Gunda, Anand L Shigli \\ Department of Pedodontics and Preventive Dentistry, Bharati \\ Vidyapeeth University, India
}

Correspondence: Anil T Patil, Department of Pedodontics and Preventive Dentistry, Bharati Vidyapeeth Deemed University, Dental College and Hospital, Sangli, Maharashtra, India, Tel 91 9850983500, Email dranilp0888@gmail.com

Received: September 23, 2016 | Published: March 23, 2017
Abbreviations: IR, intentional replantation; PDL, periodontal ligament

\section{Introduction}

Traumatic injuries occur in young people usually involving the enamel of the teeth with or without pulpal involvement and supporting structures and sometimes causing avulsion or bone fracture. Dental trauma like a crown root fracture involving enamel, dentin and cementum due to horizontal impact occurs below the gingival margin and could be classified depending on the pulpal involvement as complicated or uncomplicated. Permanent maxillary incisors well before the complete root formation are most susceptible for these injuries causing either inflammation of the pulp or necrosis. During the maturation of permanent teeth, dentoalveolar trauma may cause incomplete formation of root or resorption of root. There are many techniques that have been developed for inducing formation of the hard tissue and closure of the apex. Orthodontic treatment, extrusion by surgical method, osteotomy, gingivectomy, and intentional replantation are the treatment options of an infraosseous or subgingival fracture. ${ }^{1}$ Grossman in 1982 defined intentional replantation as follows: "a purposeful removal of a tooth and its reinsertion into the socket almost immediately after sealing the apical foramina." He also stated that it is "the act of deliberately removing a tooth and following examination, diagnosis, endodontic manipulation, and repair, returning the tooth to its original socket to correct an apparent clinical or radiographic endodontic failure". It is a one-stage treatment that will maintain the natural tooth aesthetics if successful. ${ }^{2}$ IR has undergone a sort of revival in recent years. Serious questions are raised in the field of endodontics whether it should be considered a treatment of "last resort," when intracanal or surgical endodontic treatments are not recommended, or as a conventional treatment procedure. Preservation of cellular vitality of the periodontal ligament under aseptic conditions is the most important step in any reimplantation following therapeutic extraction of a tooth or avulsion. For the survival of the tooth regeneration of the PDL is very important and if the tooth is kept outside the oral environment for more than an hour alkalosis can occur. For the normal functioning the removal of all tissue debris and irritating substances from the root surface, achievement of a good apical seal and reinforcement of the crown structure are of utmost importance. In 1961, Loe and Waerhaugtried in their study replanted the teeth immediately to keep the PDL vital. As a result, ankylosis did not occur; but, resorption was seen in all the teeth which were repaired with cementum. These results were further confirmed in 1965 by Deeb and in 1966 by Edwards. In 1968, Sherman proved that normal PDL could be kept vital. ${ }^{2}$ Intentional replantation is indicated. ${ }^{2}$

i. When there is failure or it is impossible to perform any endodontic non-surgical and surgical treatment;

ii. When the patient is unable to open mouth fully, making the performance of nonsurgical or peri-radicular surgical endodontic procedures difficult.

In the case of any sort of obstruction in the root-canals:

a. When there are non-accessible restorative or perforation root defects via the usual surgical approach without excessive loss of root length or alveolar bone.

b. It has been used in the treatment of endodontic-periodontic lesions, vertical fractures, poor prognosis of periodontally involved teeth and few anatomical malformations, like radicular groove.

c. Endodontically treated teeth with vertical root fractures.

d. Crown-root fracture of teeth

i. Hopeless teeth with periodontally involvement and

ii. Replacement resorption of teeth

Contra-indications may include, ${ }^{2}$

a. Curved and long roots;

b. Tooth mobility and periodontal diseases with poor prognosis; 
c. Multi-rooted teeth with diverging roots that make extraction and replantation impossible; and

d. Non restorable caries

e. We have presented a clinical case to epitomize the potential of IR as a viable treatment option as an alternative to extraction.

\section{Case presentation}

A 15-year-old male patient was referred to the Department of Pediatric Dentistry complaining of occasional pain on biting since one year in his upper right front tooth. He gave a history of fall two years back where he fractured his maxillary central incisor but did not undergo any treatment for the same. His medical history and extraoral examination were non-contributory. Intraoral examination revealed enamel, dentin and pulp fracture involving the incisal edge in tooth 11 along with discoloration of crown (Figure 1). Periodontal probing revealed deep narrow isolated pockets on both facial and palatal aspect of tooth 11 while other teeth showed a probing depth of non-pathologic value of 2-3 mm. Pulp sensibility tests showed negative response in teeth 11. Radiographic evaluation was done by digital intraoral periapical (IOPA) radiograph, which clearly revealed an incomplete vertical root fracture in tooth 11 (Figure 2). There was wide separation of fragments. However, adjacent teeth showed no fracture lines. Patient was explained about the treatment plan for the involved teeth which included extraction of tooth 11 followed by replacement with implant or a fixed or removable prosthesis. However, patient was not convinced for extraction of tooth 11 and wanted to save the tooth. Hence, it was decided to reconstruct the fractured fragments following surgical extraction of tooth 11 followed by immediate intentional replantation. The limitations of the procedure were explained to the patient and a written consent was obtained. Lignocaine with 1: 100000 epinephrine was administered as buccal infiltration and nasopalatine nerve block. Tooth 11 was extracted as atraumatically as possible and the two fractured fragments were also recovered (Figures $3 \& 4$ ). Granulation tissue was carefully removed from the tooth and the fragments while holding the tooth in gauze moistened with normal saline and propolis solution. The alveolar socket was also carefully curetted to remove any granulation tissue and irrigated with normal saline. The extracted tooth was inspected and confirmed of no extra fracture. Root canal treatment followed by retrograde obturation was done extraorally (Figures 5-9). The fractured fragments and the tooth were etched at the fracture margins with $37 \%$ phosphoric acid for 15 seconds, and then washed and air dried. Bonding agent was brushed over the etched fragments and light cured for 20 seconds followed by reattachment of a fragment with dual cure resin cement. The resin cement was light cured for 20 seconds. Thus, the fragments were reattached, thereby completely reconstructing the whole tooth (Figures 10,11). The root surfaces were treated with tetracycline solution for 30 seconds and then replanted in the alveolar socket in its original position. Semirigid splinting was done using a 21-gauge stainless steel orthodontic wire for 10 days (Figure 12) and a postoperative IOPA radiograph was taken. The complete procedure took 20 minutes of extra-oral time. Postoperative instructions were given which included soft diet for one week and careful brushing after every meal. Doxycycline 100mg BID was prescribed for seven days. The splint was removed after 10 days. There were no signs of periapical infection and the gingiva had healed. However, the fractured fragments had separated while removing the splint. So later post space was created using peso reamers (Figure 13). Glass fibre post was cemented (Figure 14) followed by composite crown build-up. Aesthetic management of tooth 11 was denied by the patient. So a polycarbonate crown was cemented (Figures 15-18). A regular six monthly follow up was done. After one year, there was successful healing of tooth 11 with probing depth and mobility within normal physiological limits.

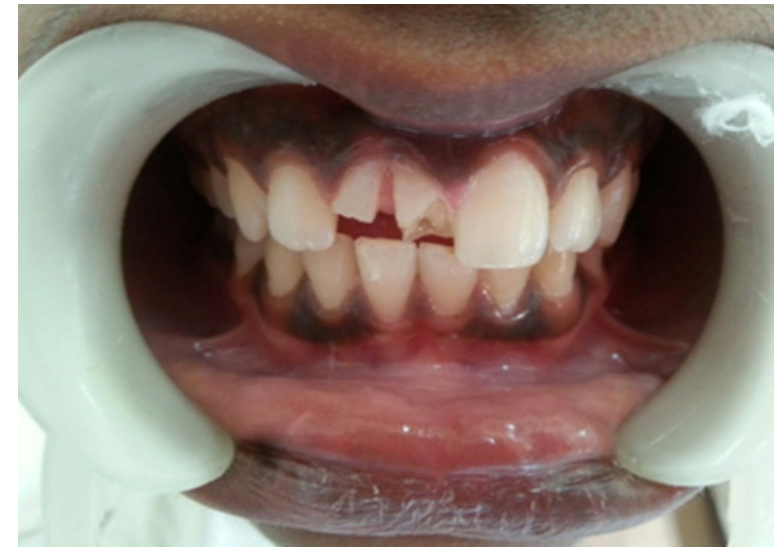

Figure I Pre-operative intra-oral photo.

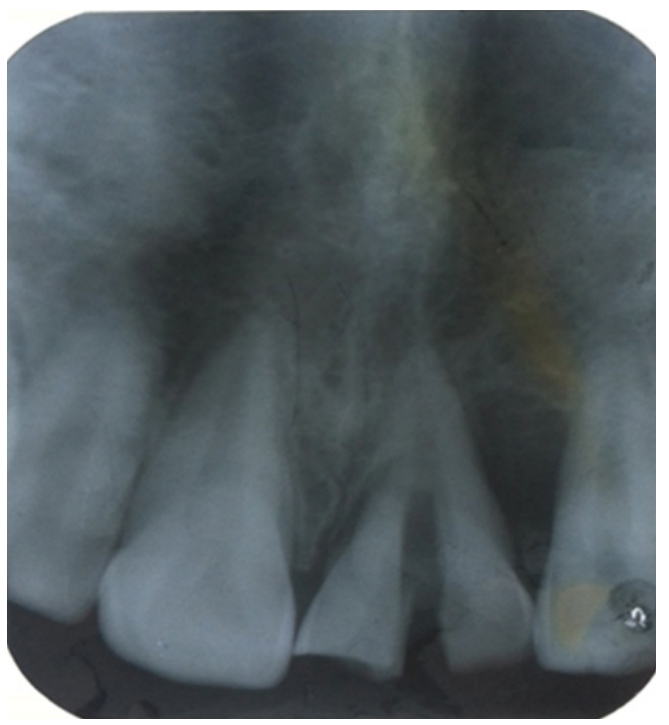

Figure 2 Pre-operative IOPA.

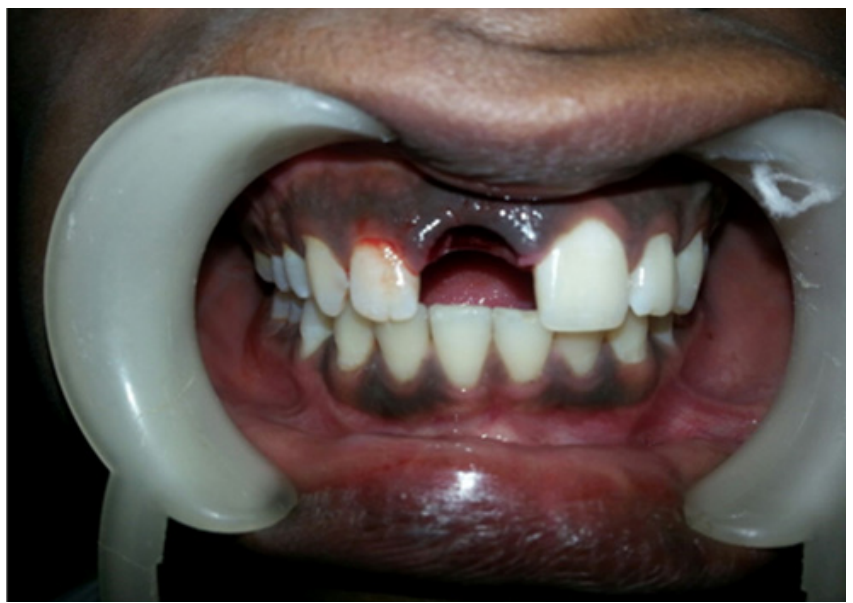




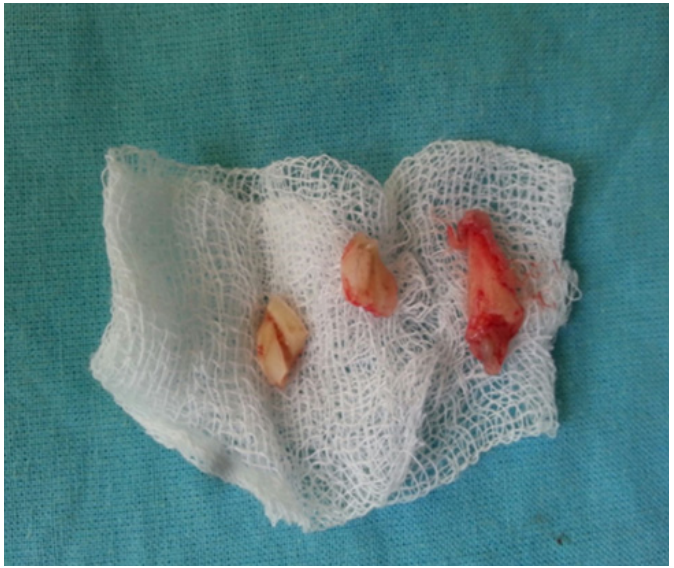

Figure 3 \& 4 Extraction of II.
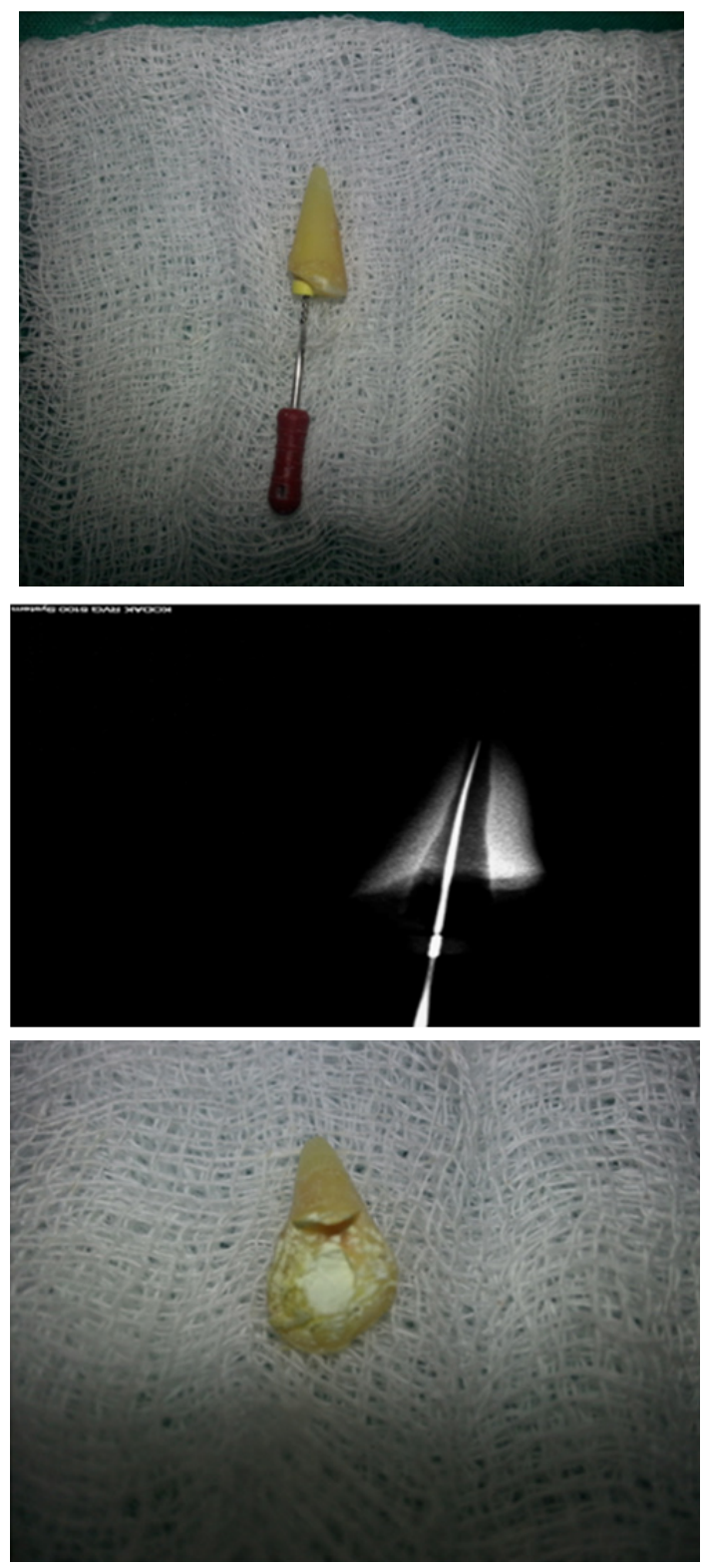
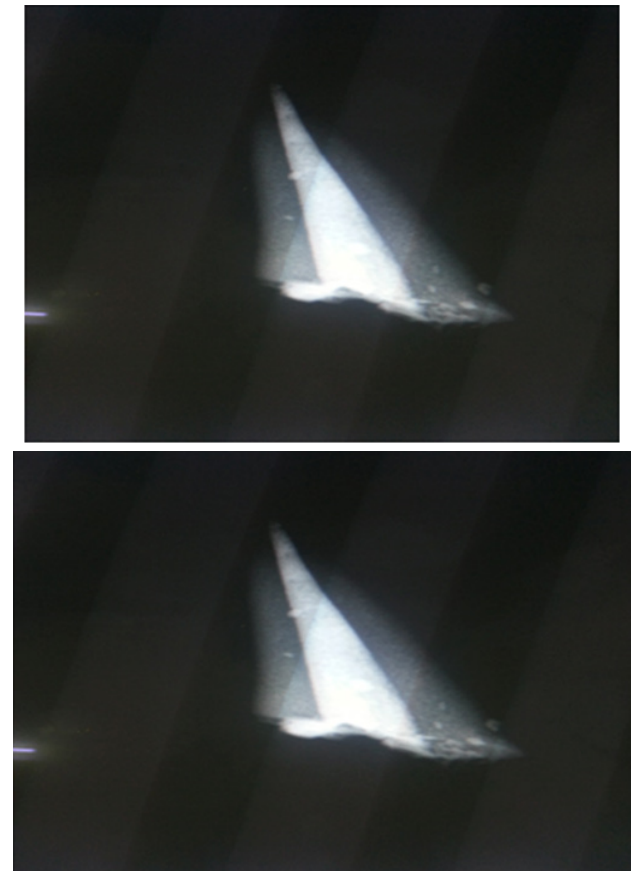

Figure 5-9 Extra-oral root canal procedure.
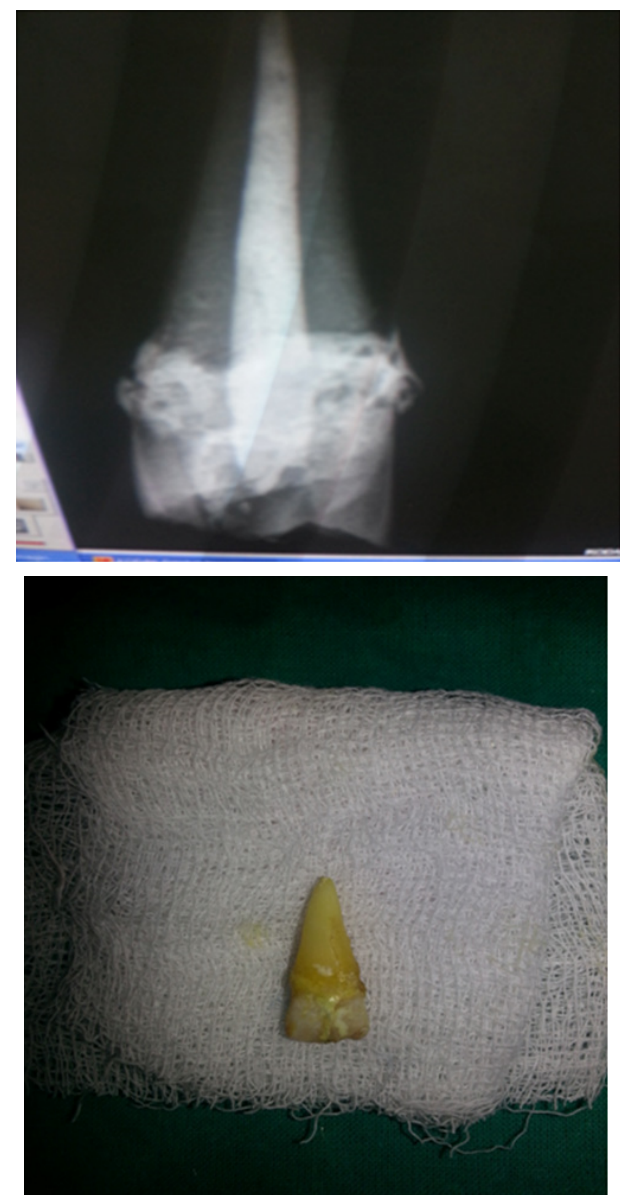

Figure I0, I I Fractured fragments bonded together. 


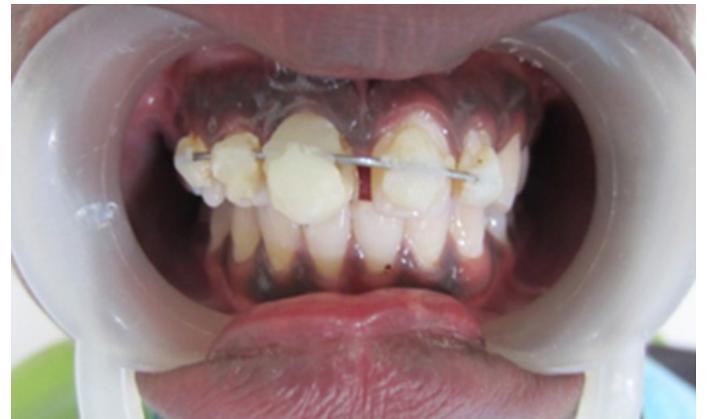

Figure 12 Splinting Done.

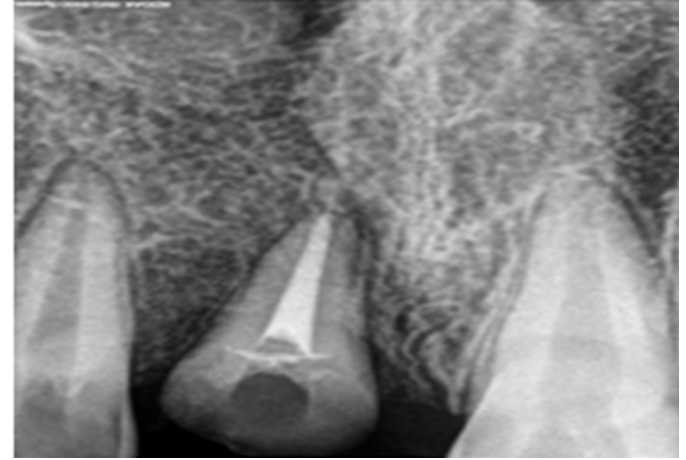

Figure 13 Post space created.

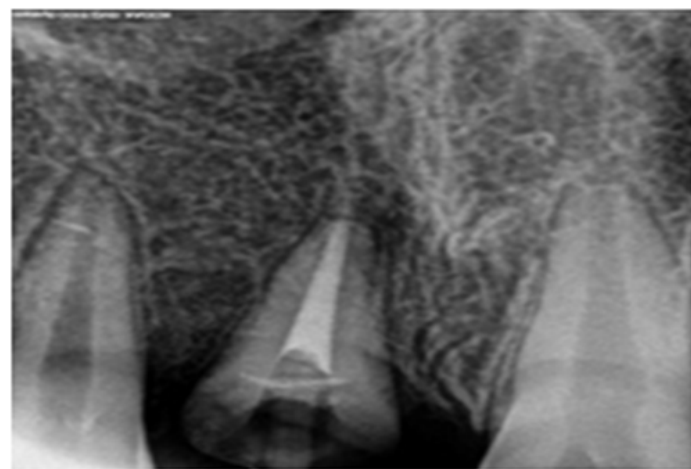

Figure 14 Glass fiber post cemented.

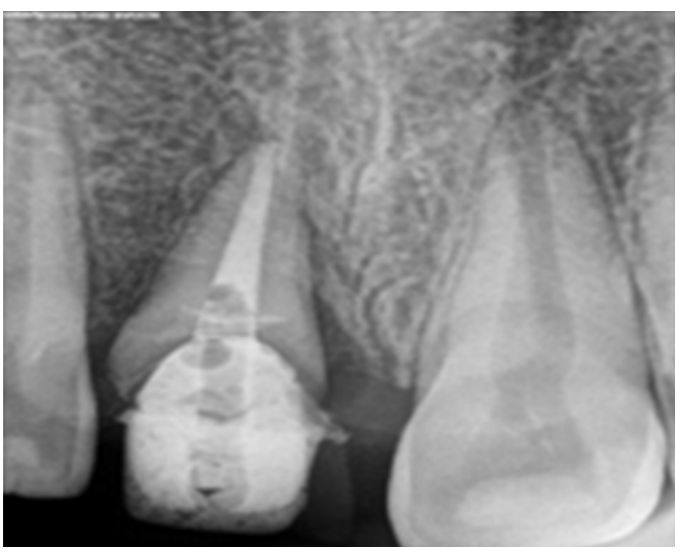

Figure 15 Radiograph of Composite core Build-up.

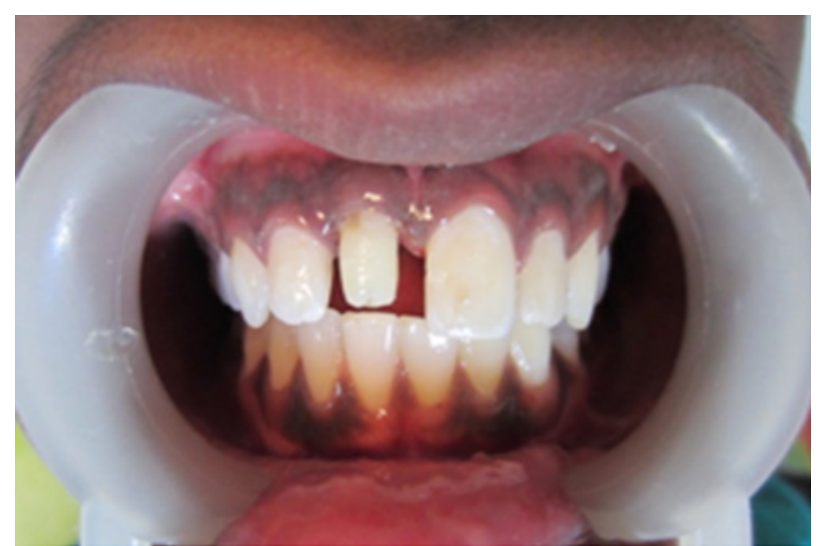

Figure 16 Clinical image of Composite core Build-up.

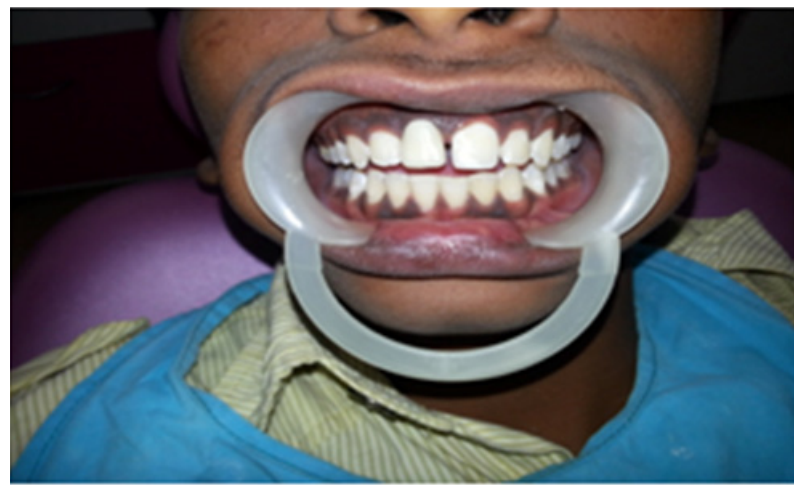

Figure 17 Polycarbonate crown cemented on II.

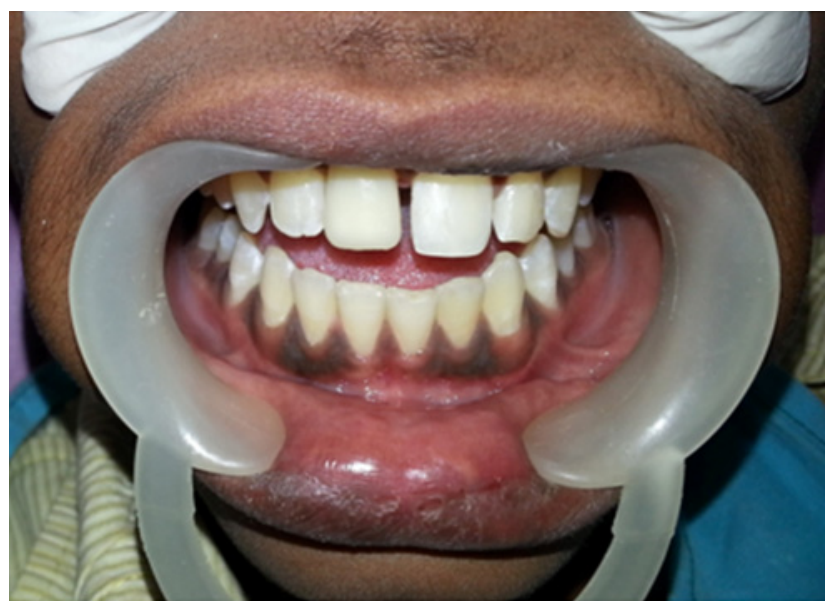

Figure I8 Polycarbonate crown cemented on II.

\section{Discussion}

Intentional replantation is an accepted endodontic procedure in cases where surgical endodontic treatment is not possible. Still not frequently used, IR is a treatment option that should be considered by dentists under such circumstances. Root resorption and ankylosis may be seen within one or two months if the standard protocols during IR are not followed. Within the first two to three years most resorptive processes are diagnosed. New resorptive processes could also rarely occur even after five or ten years. ${ }^{3}$ The success rate of IR depending on 
the periapical healing was between $72 \%$ and $89.5 \%$ in many clinical studies. Resorption of root, the persistence of apical lesion or pain, formation of periodontal pocket, and tooth mobility are reported to be the main causes of failures after IR. Complications like root cracks or fractures that occur during the extraction of teeth call for termination of the procedure. In reported IR cases root end filling materials like amalgam, zinc-oxide eugenol, glass-ionomer resin, and Mineral Trioxide Aggregate (MTA) have been used. ${ }^{4}$ It is difficult to predict the outcome for IR as various investigators have declared varying success rates. 31 cases were evaluated by Bender and Rossman with an overall success rate of $80.6 \%$ (six recorded failures). Replanted teeth survived from one day to 22 years. Second time replantation was done of a second mandibular molar that had failed after three weeks initially with no signs of failure later after 46 months of follow-up. ${ }^{5}$ Aqrabawi assessed two cases of IR and retrograde filling of mandibular second molars. In a recall visit after five years, no pathological changes were seen in the radiographs. Al-Hezaimi et al. ${ }^{6}$ in a 15 -year-old girl treated a radicular groove that predisposed her to a severe defect in the periodontium with a combination of endodontic, IR and Emdogain (Straumann) therapy. During one-year follow-up, the patient was comfortable and active healing was seen. Araujo et al. ${ }^{7}$ made it evident that root resorption, ankylosis and new attachment formation, among other processes, characterized healing of a replanted root which had been extracted and deprived of vital cementoblasts. They also made it evident that Emdogain therapy, which is, conditioning with EDTA and placement of enamel matrix proteins on the detached root surface, did not interfere with the healing process. Yu et al. ${ }^{8}$ demonstrated a case in which a combined endodontic-periodontic lesion on a mandibular first molar was treated by IR followed by application of hydroxyapatite. During the 15-month follow-up examination, the tooth was healthy both clinically and radiographically and well functioning. ${ }^{9}$ Raghoebar and Vissink replanted 29 teeth, which consisted of two mandibular first molars, 17 mandibular second molars, one mandibular third molar and nine maxillary second molars, and did follow up for 62 months. They reported a success rate of $72 \%$ and 25 of them were still in function. ${ }^{10}$ Nuzzolese and others concluded that the success rate of IR at 5 years follow up ranges from $70 \%$ to $91 \% .{ }^{11}$ To relieve continuing symptoms of a mandibular second molar Benenati reported a case of nonsurgical endodontic treatment and intentional replantation of the same. During a 15 and half year clinical follow-up patient was found to be asymptomatic, the tooth still functional, and a recall radiograph did not show any evidence of root resorption. ${ }^{12}$ Peer studied and reviewed 9 cases of IR that demonstrated the usefulness of the procedure for a variety of indications. Out of these cases only 1 case of replantation showed pathosis, followed by root resorption or ankylosis. This report concluded that IR is a reliable and predictable procedure and more often should be a treatment of choice to conserve the natural dentition. ${ }^{13}$ Demiralp and others after conditioning root surfaces with tetracycline- $\mathrm{HCl}$ of periodontally involved teeth evaluated the clinical and radiographic results of IR. Thirteen patients ( 7 women and 6 men; age range: $35-52$ years) with 15 periodontally involved "hopeless" teeth were included in this study. Tetracycline-HCL, at a concentration of $100 \mathrm{mg} / \mathrm{mL}$, was applied to the root surfaces for 5 minutes. During a 6 month follow up, radiographically no root resorption or ankylosis was seen. Therefore, IR can be an alternative treatment of choice to extraction in cases where severe periodontal destruction is found and no other treatment option can be considered. ${ }^{14}$ Majorana and others studied and followed 45 cases of dental trauma for a period of 5 years and recorded the complications and responses to treatment. In 45 cases root resorption was seen (17.24\%). Out of these, 9 were related with luxation injury (20\%) and $36(80 \%)$ with avulsion. 30 cases of inflammatory root resorption (18 transient and 12 progressive) and 15 cases of ankylosis and osseous replacement were found..$^{15}$ IR of an immature lower incisor which had a periapical lesion was performed by Shintani and others. Extraction of the lower incisor followed by curettage of the periapical lesion was done. The root canal of the tooth was then irrigated, followed by calcium hydroxide and iodoform paste filling. Thereafter the tooth was secured with an arch wire splint. Later, after five years, no abnormalities were found clinically or radiographically. The root apex was obturated by an apical bridge formation. ${ }^{16}$

\section{Conclusion}

In order to provide the best long-term prognosis for a tooth that is to be replanted intentionally, the tooth must be kept out of the socket for the shortest period possible; the extraction of the tooth should be atraumatic to minimize damage to the cementum and the PDL. The PDL attached to the root surface be kept moist in saline, Hanks' balanced salt solution, Viaspan or a doxycycline solution for the entire time the tooth is outside the socket.

\section{Funding}

None.

\section{Acknowledgements}

None.

\section{Conflicts of interest}

The authors declare that there is no conflict of interest.

\section{References}

1. Sandra Rivera, Rivail Antonio Sergio, Luciana Moura, et al. Clinical management of a complicated crown-root fracture: A Case report. Braz Dent J. 2011;22(3):258-262.

2. Muhamad Abu-Hussein, Sarafianou Aspasia, Abdulgani Azzaldeen Eight-year follow-up of successful intentional replantation. Roots, international magazine of Endodontology. 2013;9(3):1-38.

3. Viswa Chandra R, Mahalinga Bhat K. Twenty-year Follow-up of an Unconventional Intentional Replantation; JCD, September 2006;72(7):639-642.

4. Subay RK, Subay MO, Balkaya CM. Intentional Replantation of a Mandibular Canine with Multiple Iatrogenic Endodontic Complications. OHDM. 2014;13(3):811-814.

5. Bender IB, Rossman LE. Intentional replantation of endodontically treated teeth. Oral Surg Oral Med Oral Pathol. 1993;76(5): 623-630.

6. Aqrabawi J. Five-year follow-up of successful intentional replantation. Dent Update. 1999;26(9):388-390.

7. Al Hezaimi K, Naghshbandi J, Simon JH, et al. Successful treatment of a radicular groove by intentional replantation and Emdogain therapy. Dent Traumatol. 2014;20(4):226-228.

8. Araujo M, Hayacibara R, Sonohara M, et al. Effect of enamel matrix proteins ('Emdogain') on healing after the re-implantation of "periodontally compromised" roots. An experimental study in the dog. $J$ ClinPeriodontol. 2003;30(10):855-861.

9. Yu L, Xu B, Wu B. Treatment of combined endodontic-periodontic lesions by intentional replantation and by the application of hydroxyapatites. Dent Traumatol. 2003;19(1):60-63. 
10. Raghoebar GM, Vissink A. Results of intentional replantation of molars. Journal of Oral Maxillofacial Surgery. 1999;57(3): 240-244.

11. Nuzzolese E, Cirulli N, Lepore MM, et al. Intentional dental reimplantation: a case report. J Contemp Dent Pract. 2004;5(3): 121-130.

12. Benenati FW. Intentional replantation of a mandibular second molar with long-term follow-up: report of a case. Dent Traumatol. 2003;19(4):233236.

13. Peer M. Intentional replantation-a 'last resort' treatment or a conventional treatment procedure? Nine case reports. Dent Traumatol. 2004;20(1):4855 .
14. Demiralp B, Nohutcu RM, Tepe DI, et al. Intentional replantation for periodontally involved hopeless teeth. Dent Traumatol. 2003;19(1):4551.

15. Majorana A, Bardellini E, Conti G, et al. Root resorption in dental trauma: 45 cases followed for 5 years. Dent Traumatol. 2003;19(5):262-265.

16. Shintani S, Tsuji M, Toyosawa S, et al. Intentional replantation of an immature permanent lower incisor because of a refractory peri-apical lesion: case report and 5-year follow-up. Int J Paediatr Dent. 14(3):218222. 